\author{
Marquette University \\ e-Publications@Marquette
}

6-2009

\title{
Demonstration of Bias-Controlled Algorithmic Tuning of Quantum Dots in a Well (DWELL) MidIR Detectors
}

\author{
Woo-Yong Jang \\ University of New Mexico \\ Majeed M. Hayat \\ Marquette University, majeed.hayat@marquette.edu \\ J. Scott Tyo \\ University of Arizona - Tucson \\ Ram S. Attaluri \\ University of New Mexico \\ Thomas E. Vandervelde \\ University of New Mexico
}

See next page for additional authors

Follow this and additional works at: https://epublications.marquette.edu/electric_fac

Part of the Computer Engineering Commons, and the Electrical and Computer Engineering Commons

\section{Recommended Citation}

Jang, Woo-Yong; Hayat, Majeed M.; Tyo, J. Scott; Attaluri, Ram S.; Vandervelde, Thomas E.; Sharma, Yagya D.; Shenoi, Rajeev; Stintz, Andreas; Cantwell, Elizabeth R.; Bender, Steven C.; Lee, Sang Jun; Noh, Sam Kyu; and Krishna, Sanjay, "Demonstration of Bias-Controlled Algorithmic Tuning of Quantum Dots in a Well (DWELL) MidIR Detectors" (2009). Electrical and Computer Engineering Faculty Research and Publications. 590.

https://epublications.marquette.edu/electric_fac/590 


\section{Authors}

Woo-Yong Jang, Majeed M. Hayat, J. Scott Tyo, Ram S. Attaluri, Thomas E. Vandervelde, Yagya D. Sharma, Rajeev Shenoi, Andreas Stintz, Elizabeth R. Cantwell, Steven C. Bender, Sang Jun Lee, Sam Kyu Noh, and Sanjay Krishna 
Marquette University

e-Publications@Marquette

\section{Electrical and Computer Engineering Faculty Research and Publications/College of Engineering}

This paper is NOT THE PUBLISHED VERSION; but the author's final, peer-reviewed manuscript. The published version may be accessed by following the link in th citation below.

IEEE Journal of Quantum Electronics, Vol. 45, No. 6, (June 2009): 674-683. DOI. This article is (C) Institute of Electrical and Electronic Engineers (IEEE) and permission has been granted for this version to appear in e-Publications@Marquette. Institute of Electrical and Electronic Engineers (IEEE) does not grant permission for this article to be further copied/distributed or hosted elsewhere without the express permission from Institute of Electrical and Electronic Engineers (IEEE).

\section{Demonstration of Bias-Controlled Algorithmic Tuning of Quantum Dots in a Well (DWELL) MidIR Detectors}

Woo-Yong Jang Department of Electrical and Computer Engineering, University of New Mexico, Albuquerque, NM Majeed M. Hayat

Department of Electrical and Computer Engineering, University of New Mexico, Albuquerque, NM J. Scott Tyo University of Arizona, Tucson, AZ

Ram S. Attaluri

Department of Electrical and Computer Engineering, University of New Mexico, Albuquerque, NM Thomas E. Vandervelde

Department of Electrical and Computer Engineering, University of New Mexico, Albuquerque, NM Yagya D. Sharma

Department of Electrical and Computer Engineering, University of New Mexico, Albuquerque, NM 


\title{
Rajeev Shenoi
}

Department of Electrical and Computer Engineering, University of New Mexico, Albuquerque, NM Andreas Stintz

Department of Electrical and Computer Engineering, University of New Mexico, Albuquerque, NM Elizabeth R. Cantwell

Los Alamos National Laboratory, Los Alamos, NM

Steven C. Bender

Los Alamos National Laboratory, Los Alamos, NM

Sang Jun Lee

Center for Advanced Measurement and Instrumentation, Korea Research Institute of Standards and Science, Daejeon

Sam Kyu Noh

Korea Research Institute of Standards and Science, Daejeon

Sanjay Krishna

Department of Electrical and Computer Engineering, University of New Mexico, Albuquerque, NM

\begin{abstract}
:
The quantum-confined Stark effect in intersublevel transitions present in quantum-dots-in-a-well (DWELL) detectors gives rise to a midIR spectral response that is dependent upon the detector's operational bias. The spectral responses resulting from different biases exhibit spectral shifts, albeit with significant spectral overlap. A postprocessing algorithm was developed by Sakoglu that exploited this bias-dependent spectral diversity to predict the continuous and arbitrary tunability of the DWELL detector within certain limits. This paper focuses on the experimental demonstration of the DWELL-based spectral tuning algorithm. It is shown experimentally that it is possible to reconstruct the spectral content of a target electronically without using any dispersive optical elements for tuning, thereby demonstrating a DWELL-based algorithmic spectrometer. The effects of dark current, detector temperature, and bias selection on the tuning capability are also investigated experimentally.
\end{abstract}

\section{Keywords}

IEEE Keywords, Quantum dots, Detectors, Optical tuning, Laboratories, Optical sensors, Spectroscopy, Temperature sensors, Materials science and technology, Dispersion, Educational technology

\section{SECTION I. Introduction}

IR spectral imaging continues to attract more and more interest as it offers a powerful solution to a wide range of challenging problems in remote sensing. These include unveiling concealed objects or targets, identifying and classifying chemical or biological compounds, and monitoring environmental changes, only to name a few. In a conventional imaging spectrometer, the spectral information in a scene is captured by sensing a wide range of narrow segments of the IR spectrum at every pixel, with each segment constituting a spectral band. This is typically achieved by using dispersive optics (or possibly interferometric methods) that allow confinement of the spectral content of the signal prior to photodetection. Different types of detectors, each sensitive to a prescribed spectral range, may additionally be utilized to cover a wider spectral range. 
In recent years, the long-wave IR (LWIR: 8-12 $\mu \mathrm{m}$ ) region of the electromagnetic spectrum has been particularly of great interest to remote sensing. Targets close to room temperature are dominated by emitted radiation over reflected radiation in the LWIR region, and they can be sensed by their apparent temperatures and spectral signatures in the LWIR. Currently, there are three main material technologies for photonic IR photodetectors in the LWIR region. The HgCdTe (MCT) detector is the current state of the art due to its high responsivity and detectivity; however, there are well-known problems with this material, especially those pertaining to its epitaxial growth. The presence of large interface instabilities, and etch-pit and void-defect densities cause large uncertainties and pixel-to-pixel fluctuations in detectivity [1]. A second possible approach for LWIR detection is to apply type-II strained-layer superlattice (SLS) with material combinations like InAsSb-InSb or InGaSbInAs [2], [3]. The epitaxial growth technique for the antimonides (Sb) is also not very mature due to group $\mathrm{V}$ intermixing during growth. Surface passivation in processing is another concern for Sb-based devices. Nonetheless, SLS seems to be a promising technology for LWIR detection.

The third approach for IR detection is to exploit intersublevel transitions in quantum-confined heterostructures such as quantum well IR photodetectors (QWIPs) using III-V compounds [4]-[5][6]. Potential advantages of QWIP focal plane arrays (FPAs) include a lower uncorrected-response nonuniformity (typically $1 \%-3 \%$ ) coupled with a higher operability (above 99.9\%) compared to MCT detectors [7], and more mature growth and manufacturing III-V technology. In the past few years, normal-incidence midIR detection has been demonstrated with intersublevel transitions in self-organized quantum dots (QDs) [8], [9]. Longer intersublevel relaxation time in the dots could also produce a significant increase in device detectivity [10]. Additionally, since the ground state is lowered with respect to GaAs band edge in device structure, we expect lower dark current levels in QD detector as compared to earlier quantum well (QW) detectors. This reduced thermionic emission can lead to higher operating temperatures, thereby reducing the complexity of associated cryocoolers.

Furthermore, InAs/InGaAs quantum-dots-in-a-well (DWELL) photodetectors [11] have also been developed in order to optimize the performance of the aforementioned QD detector.

In our group at the Center for High Technology Materials (CHTM) at the University of New Mexico, the DWELL detector has been designed and fabricated for both its potential for high sensitivity and its bias-controlled tunability [12]. (The DWELL detector is also inherently sensitive to normally incident photons.) Structurally, the active region of the DWELL detector is composed of a combination of InAs QDs and less strained InGaAs-GaAsAIGaAs QWs. A key feature of the DWELL detector is that its responsivity can be altered by varying the well width and material systems, which change the intersublevel transitions between energy levels (i.e., dot-to-dot, dot-to-well, and dot-continuum (barrier) transitions) in a DWELL structure [11]. More importantly to the present paper, the quantum-confined Stark effect (QCSE), resulting from dots in an asymmetric well, allow the spectral response of DWELL sensor to change continuously as the applied bias voltage is varied incrementally [13]. (It is to be noted that the QCSE in interband transitions and electroabsorption in QW structures have been studied by Miller et al. [17] and Aivaliotis et al. [18].) Hence, a single DWELL detector can serve as multiple detectors with different spectral responses. For example, a DWELL-based FPA was recently fabricated at CHTM successfully demonstrating two-color or multicolor operation in midwave IR (MWIR, 3-5 $\mu \mathrm{m}$ ) and LWIR regions [14][15][16].

DWELL structures have been grown that exhibit a continuous shift in their spectral response as the applied bias is varied, albeit with significant spectral overlap. To exploit this bias-controlled spectral diversity offered by the DWELL detector, a theoretical effort was launched by our group to develop an algorithmic, continuous spectraltuning strategy [19], [20]. This approach combines the electrically yet broadly tunable DWELL detector with a signal processing technique to allow both the center wavelength (so-called tuning wavelength) and the spectral resolution to be independently selected in MWIR and LWIR regions. In this paper, we report on the experimental demonstration of the concept of DWELL-based algorithmic tuning and further develop an algorithmic 
spectrometer. The performance of DWELL-based algorithmic spectrometer (DAS) is examined taking into account issues such as sensitivity to bias selection, dark current, and temperature.

\section{SECTION II. DWELL Detector}

\section{A. Device Description and Principle of Operation}

A DWELL detector is basically a hybrid of conventional QW and QD detectors. In a representative DWELL heterostructure, InAs QDs are embedded in InGaAs-GaAs multiple QW structures and electrons in the ground state of QD are promoted to a set of bound states within the QW by photoexcitation. Altering the QW thickness of the DWELL detector alters the nature of the allowable energy transitions (bound-to-bound, bound-to-quasibound, and bound-to-continuum), thereby altering the DWELL's operating wavelengths. These energy transitions enable the detection of photons from MWIR to VLWIR within a single detector. Moreover, a biasdependent spectral response is also observed in DWELL detectors due to the QCSE. The asymmetric geometry of the electronic potential, due to the shape of the dot and the different thicknesses of QW above and below the dot, results in variation of the local potential as a function of the applied bias. From these measurements, one can observe the multicolor capability of the DWELL detector structure.

The DWELL structures considered in this paper were grown by molecular beam epitaxy (MBE) and fabricated using standard lithography technique in a class 100 clean-room environment. Details of these structures are found in [11].

The detector identified here as DWELL-1780 was fabricated based on the growth and device processing techniques described before. This detector was designed to be bias-tunable and operate in the LWIR region. By optimizing the device structure of DWELL-1780, an optimized DWELL, termed DWELL-1781, was designed and fabricated for operation at longer wavelengths. The modification in DWELL-1781, compared to DWELL-1780, included change in the shoulder width of GaAs well from 15 to $30 \mathrm{rmAA}$, which led to a lowering of the QW state and introduce a redshift in the spectral response. The growth conditions of both detectors are described in the corresponding device schematics, shown in Fig. 1. Device characterization results are depicted and discussed in the next section. Both DWELL-1780 and DWELL-1781 detectors are later employed by the proposed algorithmic spectrometer. 


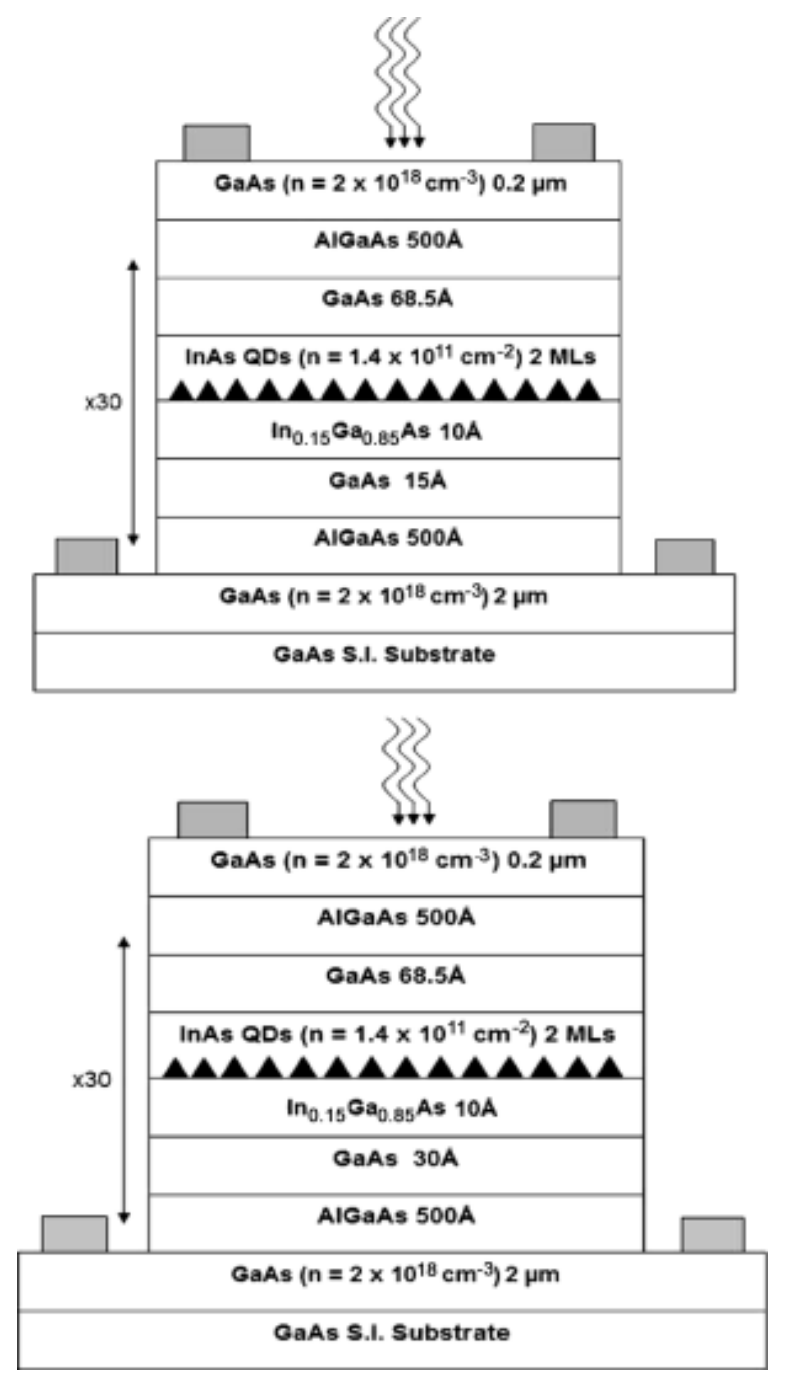

Fig. 1. Growth schematics of (top) DWELL-1780 and (bottom) DWELL-1781.

\section{B. DWELL Device Characterizations}

Spectral responses of the two DWELL devices were measured by using a Nicolet 870 FTIR spectrometer and a Keithley 428 current amplifier, which is used to control the bias applied to the detectors. Photocurrents and corresponding dark currents at different biases were also obtained using an HP oscilloscope. Bias-dependent spectral responses of the DWELL-1780 are shown in Fig. 2, which illustrates the multicolor attribute of the DWELL in the LWIR range. Fig. 2 also demonstrates photocurrent characteristic measured from a DWELL detector at different biases. The spectral measurements of the optimized DWELL-1781 are illustrated in Fig. 3. There are two distinct peaks at LWIR region observed, one in about $9.5 \mu \mathrm{m}$ and the other in $10.5 \mu \mathrm{m}$. Improvement in the performance was evident showing shifts in the operating wavelengths. The peak operating wavelengths for negative biases shift from 8.5 (in DWELL-1780) to $9.5 \mu \mathrm{m}$ (in DWELL-1781), and for positive biases, they shift from 10 (in DWELL-1780) to $10.5 \mu \mathrm{m}$ (in DWELL-1781). This redshift is due to the lowering of the QW state in the heterostructure. 

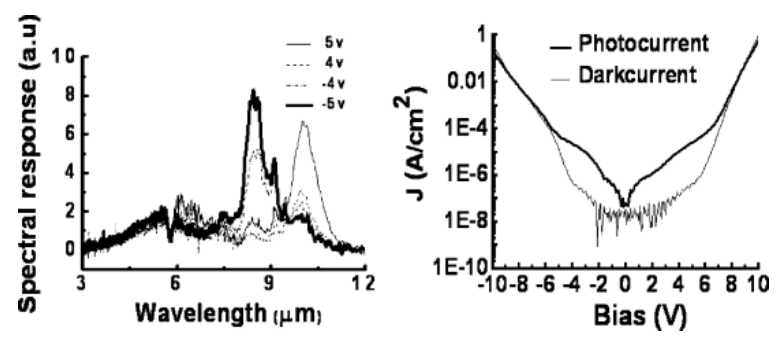

Fig. 2. (Left) Bias-dependent DWELL-1780 spectral responses at $30 \mathrm{~K}$. (Right) Photocurrent characteristic obtained from a detector at different biases.
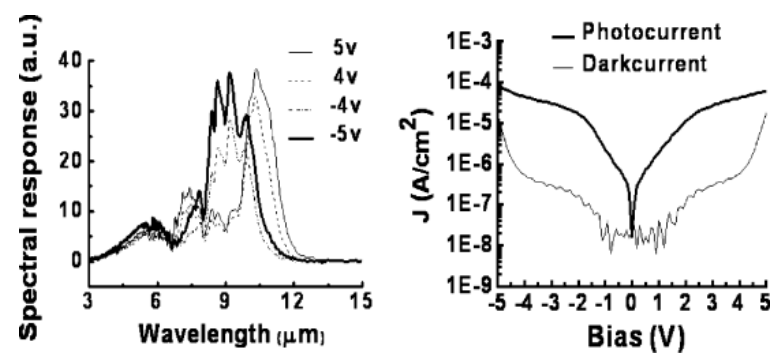

Fig. 3. (Left) Bias-dependent spectral responses of the optimized version DWELL-1781 at $30 \mathrm{~K}$. (Right) Its photocurrent characteristic.

The limitation of the DWELL's operating temperature was observed due to higher dark current levels at higher device temperatures. Fig. 4 shows the bias-dependent spectral responses for various device operating temperatures. It is to be noted that the performance of DWELL-1781 begins to degrade dramatically as device operating temperature exceeds $60 \mathrm{~K}$. At $77 \mathrm{~K}$, noise dominates the spectral measurements and almost no spectral variation is observed for any bias.
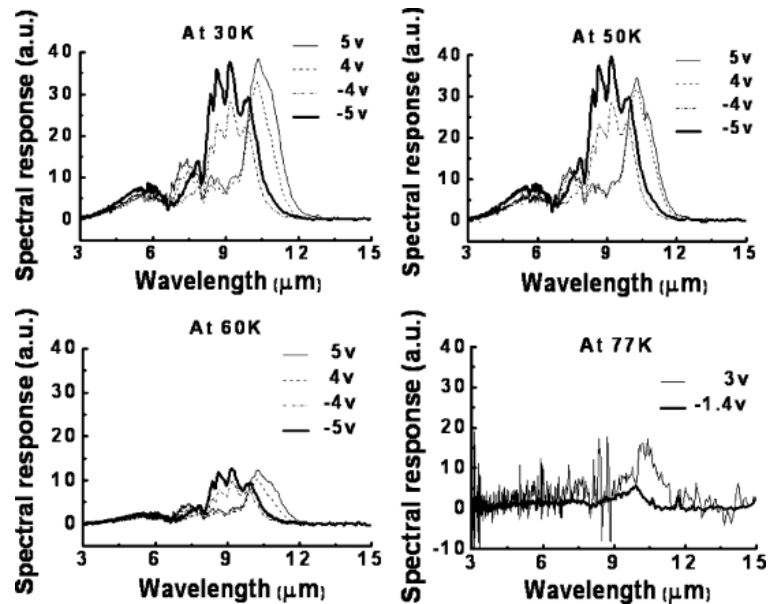

Fig. 4. Bias-dependent spectral responses of DWELL-1781 as a function of a number of operating temperatures at (top left) $30 \mathrm{~K}$, (top right) $50 \mathrm{~K}$, (bottom left) $60 \mathrm{~K}$, and (bottom right) $77 \mathrm{~K}$.

More structural adjustments were made to improve the operating temperature of DWELL-1781, yielding DWELL2299. Notable differences between these two devices are the incremental change in the shoulder size of the GaAs well (from 30 rmAA in DWELL-1781 to 40 rmAA in DWELL-2299) and the presence of shoulders on both sides of the InGaAs layer giving rise to a double DWELL (D-DWELL) design [21]. Fig. 5describes the performance of DWELL-2299, demonstrating the bias-dependent spectral responses at $77 \mathrm{~K}$, which is much higher than the maximum operating temperature of DWELL-1781. 

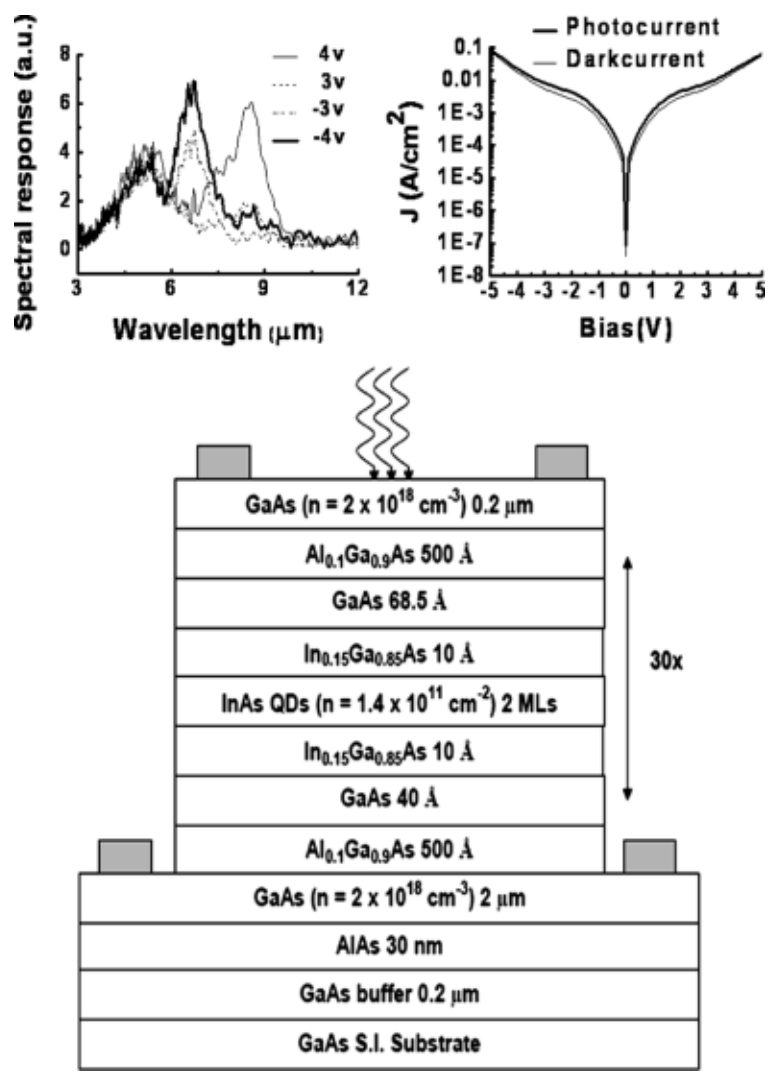

Fig. 5. Bias-dependent spectral responses of DWELL-2299 at (top left) $77 \mathrm{~K}$ and (top right) photocurrent characteristic. Bottom: growth schematic of a device.

Intersublevel transitions in conduction band diagram are depicted in Fig. 6. Based on band offsets from the photoluminescence (PL) measurements of DWELL-2299, two MWIR peaks each at 5.25 and $6.5 \mu \mathrm{m}$ are due to a bound-to-bound transition between the ground state of the QD and the states within the GaAs QW. The LWIR peak at $8.35 \mu \mathrm{m}$ is due to a transition between the ground state of the QD and the state within the InGaAs QW [22]. 

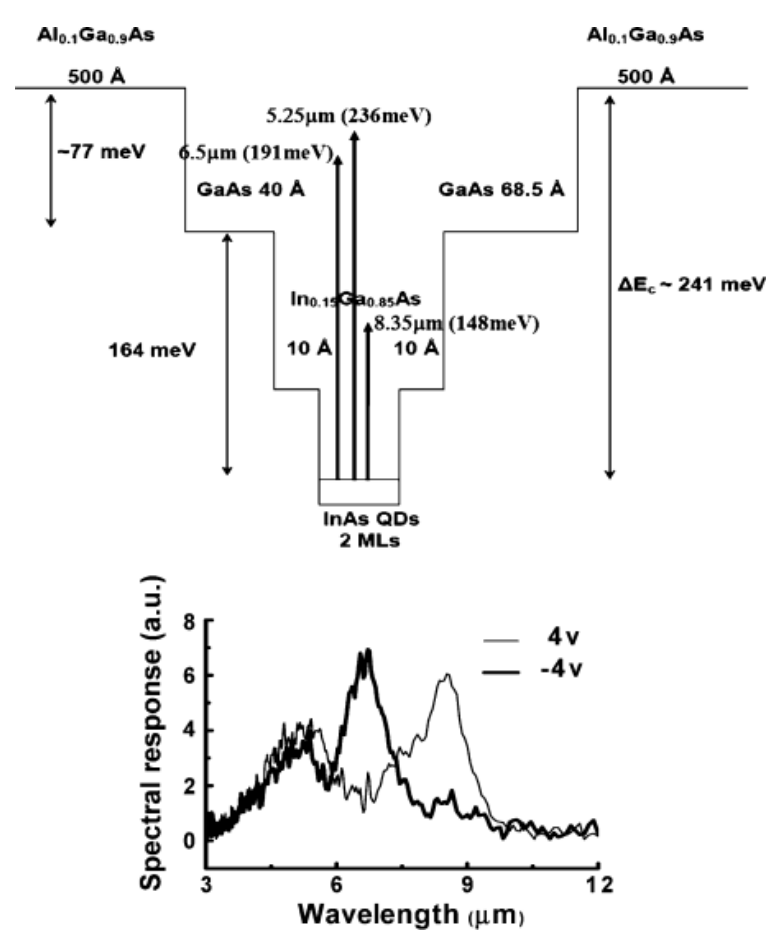

Fig. 6. (Top) Energy transition levels in the conduction band and (bottom) the corresponding peak wavelengths at the spectral responses of DWELL-2299 at $77 \mathrm{~K}$.

\section{SECTION III. Algorithmic Spectrometer}

We begin by providing a brief, qualitative description of the algorithmic spectrometer. A mathematical description is given in Section III-A, followed by the experimental verification of the algorithmic spectrometer in Section III-B.

We will assume that an unknown target is probed repeatedly by the DWELL detector, each time using a different operating bias, resulting in a set of bias-dependent photocurrents. The idea of an algorithmic spectrometer is to utilize these bias-dependent photocurrents to construct an approximation of the spectrum of the object of interest without using any physical spectrometer or optics. The algorithmic spectrometer is implemented as follows. First, a hypothetical narrowband tuning filter is selected with a specified center (tuning) wavelength and a specified full-width at half-maximum (FWHM) linewidth. Next, a set of weights, one for each operating bias, is obtained through the use of the projection algorithm reported in [20]. These weights have the property that once used to form a weighted linear superposition of the DWELL's bias-dependent spectral responses, the resulting superposition spectral response best approximates the hypothetical tuning filter [20]. Next, the biasdependent photocurrents are linearly combined using the same weights, yielding an approximation of target's spectrum at the prescribed tuning wavelength. The reconstruction of the target's spectrum is completed by repeating the earlier procedure for other tuning wavelengths.

\section{A. Review of the Spectral Tuning Algorithm}

We begin by reviewing germane aspects of the spectral-tuning algorithm [19], [20] to be used with the DWELL detector in producing the DAS. Consider an arbitrary object of interest whose transmittance in the LWIR range is represented by the function $f(\lambda)$. We assume that the object is illuminated by a blackbody source. Suppose that a DWELL detector is used to probe the illuminated object using various biases, $v_{1}, \ldots, v_{K}$, yielding a set of biasdependent photocurrents, $I_{1}, \ldots, I_{K}$, respectively. Let the detector's spectral response at the $i$ th applied bias be denoted by $R_{i}(\lambda)$. 
Our approach for achieving an algorithmic spectrometer can be described as follows. Imagine an ideal (and hypothetical) narrowband LWIR tuning filter centered at wavelength $\lambda_{n}$ and with transmittance function $r\left(\lambda ; \lambda_{n}\right)$. (In a conventional spectrometer, as schematically shown in Fig. 7(a), such a filter would be used with a broadband detector to estimate the spectrum of the object of interest at wavelength $\lambda_{n}$.) Our earlier theoretical work [20] provides a method for calculating a set of superposition weights, $w_{n, 1}, \ldots, w_{n, K}$, which depends upon the choice of $r\left(\lambda ; \lambda_{n}\right)$, so that the synthesized photocurrent $\hat{I}_{\lambda_{n}} \equiv w_{n, 1} I_{1}+\cdots+w_{n, K} I_{K}$, best approximates the ideal photocurrent $I_{\lambda_{n}}$ that we would have obtained if we were to sense the same target of interest $f$ using an ideal broadband (spectrally flat response) detector that is looking at the object through the spectral filter $r\left(\lambda ; \lambda_{n}\right)$. The formula for these weights is given in [20] and is repeated for convenience in the Appendix. The approximation is in the sense of minimizing the mse between the synthesized photocurrent $\hat{I}_{\lambda_{n}}$ and the ideal response $I_{\lambda_{n}}$. Our earlier theoretical work also teaches us that the same set of weights is characterized as that for which the superposition spectrum, $\hat{r}\left(\lambda ; \lambda_{n}\right) \equiv w_{n, 1} R_{1}(\lambda)+\cdots+w_{n, K} R_{K}(\lambda)$, best approximates the imaginary tuning filter $r\left(\lambda ; \lambda_{n}\right)$ in the sense of minimizing the wavelength-integrated mse. Now, if we repeat the earlier procedure while sweeping the center wavelength $\lambda_{n}$ of the narrowband tuning filter $r$ in a specified range of interest, we will obtain, for each center wavelength, an estimate of the spectrum of the source transmittance. Hence, as we sweep across the center wavelength $\lambda_{n}$ of our "hypothetical" tuning filter $r$ and apply the superposition procedure described earlier, we will reproduce the transmittance function $f$, albeit, within the confines of the approximation.

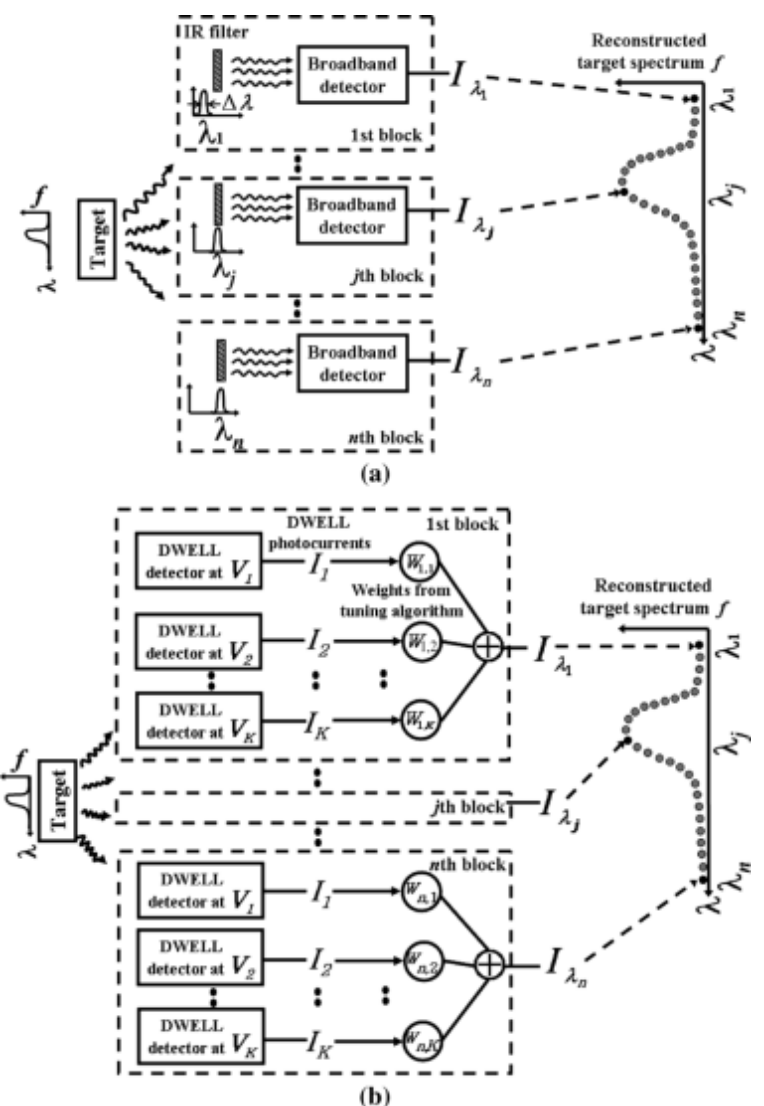

Fig. 7. (a) Conventional spectral sensing method using a standard broadband IR detector and a family of optical IR filters. (b) Proposed algorithmic spectrometer equivalent of (a). Initially, several photocurrents (of the target spectrum $f$ ) are taken at different bias voltages $v_{1} \cdots v_{k}$. Then, the measured responses at each bias are algebraically combined with predetermined weights $w_{i, n}$ that are used to match a desired filter centered at wavelength $n$. By changing the weights, the effect of different desired filters [similar to the ones used in (a)] is synthesized, albeit, without the use of any optical filters. 
The earlier concept of an algorithmic spectrometer is schematically shown in Fig. 7(b). After several repetitions for desired tuning centers, $\lambda_{1}, \ldots, \lambda_{m}$, say, the set of synthesized outputs $\hat{I}_{\lambda_{1}}, \ldots, \hat{I}_{\lambda_{m}}$ is generated and regarded as the approximate reconstruction of the spectrum of the target of interest within the prescribed wavelength range. We reiterate that these synthesized outputs approximate those obtained using an ideal IR detector in conjunction with an actual tunable spectral filter shown schematically in Fig. 7(a). Thus, the algorithmic spectrometer shown in Fig. 7(b), which uses no physical spectral filters, is functionally equivalent to the actual spectrometer schematically shown in Fig. 7(a).
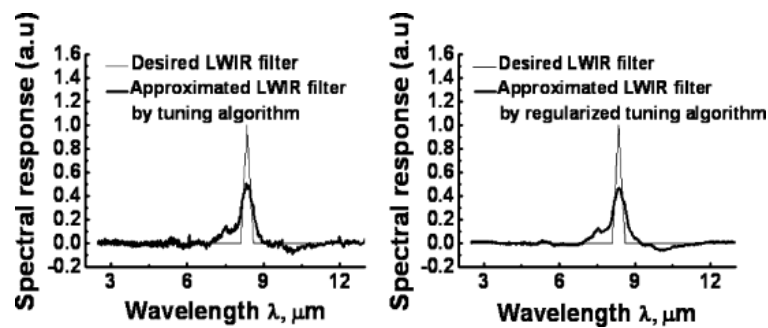

Fig. 8. Approximation of a desired narrowband LWIR tuning filter by DWELL-1780 spectra at $30 \mathrm{~K}$ with (left) $\alpha=0$ and (right) $\alpha=12$.

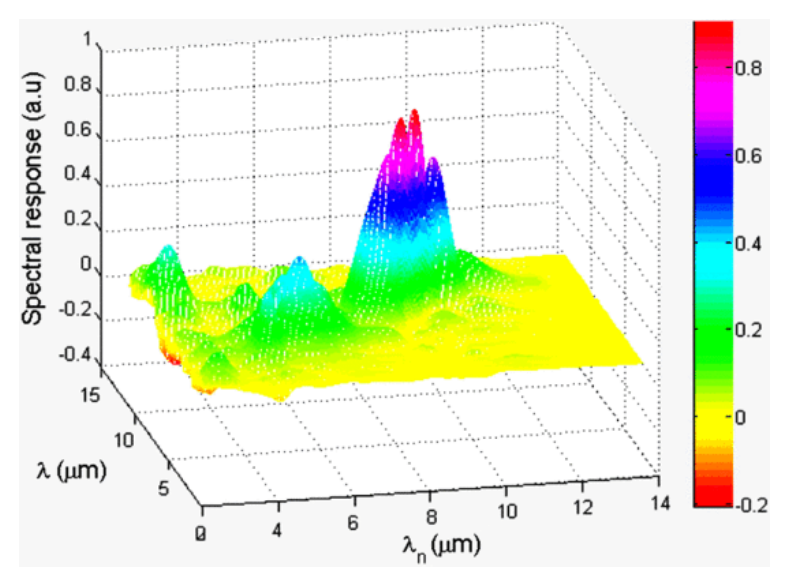

Fig. 9. Approximation of a desired tuning filter for various tuning wavelengths $\lambda_{n}$ (from 2.55 to $12.25 \mu \mathrm{m}$ ) with regularization parameter $\alpha=12$.

Before we proceed with the experimental verification of the DAS, we will show a representative example of the superposition tuning filter $\hat{r}\left(\lambda ; \lambda_{n}\right)$ that approximates a desired triangular tuning filter with center wavelength of $8.5 \mu \mathrm{m}$ and an FWHM width of $0.5 \mu \mathrm{m}$. The results corresponding to DWELL-1780 are shown in Fig. 8 using the bias-dependent spectral responses shown in Fig. 2. Note that the initial choice of $\alpha=0$ (no regularization), shown in Fig. 8(a), yielded a somewhat fluctuating reconstruction, which can be avoided. Much better results are obtained when regularization is used; for example, the choice $\alpha=12$ produced excellent results, as shown in Fig. 8(b). Generally, if the parameter $\alpha$ is selected too large, then the reconstructed spectrum loses resolution; on the other hand, if $\alpha$ is selected too small, then the reconstructed spectrum exhibits spurious fluctuations. The 3-D graph in Fig. 9 illustrates the desired tuning filter approximations over various tuning wavelengths $\lambda_{n}$ ranging from 2.55 to $12.25 \mu \mathrm{m}$, in steps of $0.05 \mu \mathrm{m}$. Good approximations $\hat{r}\left(\lambda ; \lambda_{n}\right)$ of $r\left(\lambda ; \lambda_{n}\right)$ were observed in LWIR in the range 8-10.5 $\mu \mathrm{m}$, showing the reconstructed peak regions of the desired tuning filter. However due to the effect of weak spectral responses from DWELL1780 in the MWIR region (along with atmospheric absorptions), poor approximations in the range 2.55$7 \mu \mathrm{m}$ are observed. Also the limitation of tuning filter approximation was evident beyond $11.5 \mu \mathrm{m}$ because of 
the lack of spectral responses (contents) by DWELL-1780 in Fig. 2 (left). As a result, we anticipate that the DAS with DWELL-1780 can potentially perform well over the range 8-10.5 $\mu \mathrm{m}$.

\section{B. Experimental Demonstration of DAS}

For the experiments presented here, we considered four different LWIR targets, $f_{1}(\lambda), \ldots, f_{4}(\lambda)$, as depicted in Fig. 10 (solid curves), with different center wavelengths in the range 8-10 $\mu \mathrm{m}$ and different spectral bandwidths, $\Delta \lambda=1.0-3.5 \mu \mathrm{m}$. The spectral response of the DWELL-1780 detector was measured at detector operating temperature of $30 \mathrm{~K}$ for 82 bias voltages between -5 and $5 \mathrm{~V}$ by using an FTIR spectrometer and a blackbody source. Next, for every applied bias, the photocurrent and associated dark current were measured for each one of the four targets illuminated by the global source. It is to be noted that in the experiment, the same detector was sequentially biased to generate the bias-dependent spectral response of the DWELL detector.
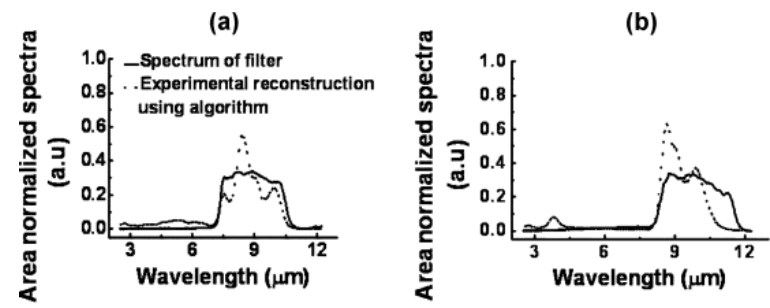

(c)
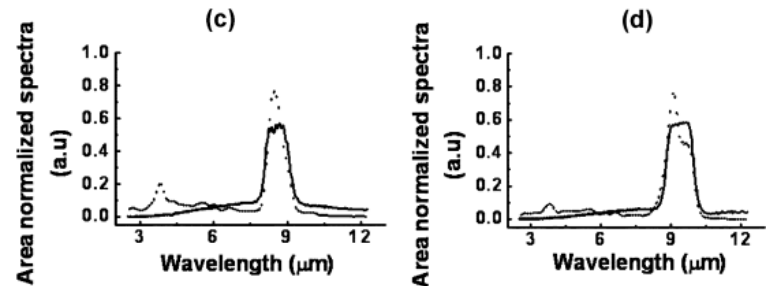

Fig. 10. Experimental reconstructions using algorithmic spectrometer incorporated with DWELL-1780 detector at 30 K. Solid curves represent the actual responses of the targets and the dotted curves represent the reconstructed spectra using tuning algorithm. (a) $f_{1}(\lambda)$. (b) $f_{2}(\lambda)$. (c) $f_{3}(\lambda)$. (d) $f_{4}(\lambda)$.

The SNR at each bias was estimated by utilizing a standard Poisson approximation to model the dark current [23], [24] in conjunction with our experimental data for the variance of the dark current. Each quantity $\mathrm{SNR}_{k}$ (corresponding to the kth bias) was calculated using

$\mathrm{SNR}_{k}=\frac{y_{p, k}}{\sigma_{N, k}}(1)$

where $y_{p, k}$ is the experimentally averaged photocurrent (over 100 realizations) and $\sigma_{N, k}$ is the standard deviation of the dark current, also calculated empirically from the dark current realizations. This step allows us to determine the noise-equivalent matrix $\Phi$, as shown by $(3)$ in the Appendix.

Next, the algorithmic tuning procedure described earlier in Section III-A was followed to calculate the synthesized superposition photocurrents, one for each desired tuning wavelength. We used 195 ideal triangular tuning filters representing $r\left(\lambda ; \lambda_{n}\right)$ (with FWHM of $0.5 \mu \mathrm{m}$, similar to the one shown in Fig. 8), whose center wavelengths range from 2.55 to $12.5 \mu \mathrm{m}$ in steps of $0.05 \mu \mathrm{m}$, and calculated the corresponding weight vectors for each center wavelength according to $(3)$ in the Appendix. As a result, 195 synthesized photocurrents are calculated according to $(2)$ in the Appendix, yielding a reconstruction of each target spectrum. The best regularization parameter of $\alpha=12$ was obtained by trial and error and used. 
Fig. 10 shows the experimentally reconstructed spectra from the DWELL-1780 detector (dotted curves) along with the actual spectra of the targets (solid curves). The figure demonstrates two key points. First, the experimental reconstructions of the target spectra are good approximations of their true spectra, validating our approach. Second, the limitation of the proposed approach is also evident. For example, the DWELL-1780 detector does not accurately reconstructed the long wavelength edge of target $f_{2}(\lambda)$ [Fig. 10(b)]. This is due to the lack of response of the DWELL-1780 detector beyond $11.5 \mu \mathrm{m}$.

We observe that, in general, the algorithmic spectrometer works well for tuning wavelengths in the spectral range (8-12 $\mu \mathrm{m})$ for which the DWELL's response is strong, as shown in Fig. 2. However, as the tuning wavelength is extended beyond $3 \mu \mathrm{m}$ (toward near IR) or beyond $12 \mu \mathrm{m}$ (toward very long wavelength IR), the tuning algorithm can no longer reconstruct the tuning filter properly, as seen in Fig. 9. This is primarily attributable to the weak response of the DWELL at these extreme wavelengths, as seen in Fig. 2. Consequently, we expect the performance of the algorithmic spectrometer also to be poor at these extreme wavelengths. Moreover, the sensitivity to extreme wavelengths is particularly accentuated in cases for which the SNR of the photocurrent is low $(<10 \mathrm{~dB})$. Thus, there is a tradeoff between the SNR and the spectral range of the algorithmic spectrometer. We therefore expect DAS to exhibit higher sensitivity to SNR at extreme wavelengths, which, in turn, would require lower operating temperatures. Additional theoretical analysis on tuning limitation is described in [19] and [20].

\section{SECTION IV. Experimental Performance Analysis}

In the previous section, we demonstrated the capability of the proposed DAS (with DWELL-1780) in the spectral sensing various LWIR targets. In this section, we study the performance of the DAS (using DWELL-1781) as a function of SNR, detector's temperature variation, and bias diversity. Here, we use the DWELL-1781, instead of using DWELL-1780 and DWELL-2299, due to its higher operating temperature, its spectral response at longer wavelengths, and its superior tunability.

We begin by examining the dependence of the DAS performance on the photocurrent's SNR. The spectrumreconstruction procedure used to generate Fig. 10(c) for the LWIR target $f_{3}(\lambda)$ was repeated for various levels of the photocurrent's SNR. For each level of the SNR, a scaling factor $\rho$ is used to modify the average photocurrent $y_{p, k}$ uniformly in $k$, which, in turn, amplifies the photocurrent's SNR according to $\underline{1}$ ). (The noise variance in (1) is held fixed.) This analysis is useful, for instance, in examining the effect of changing the quantum efficiency of the QDIP on the performance of the algorithmic spectrometer. The results are shown in Fig. 11; they show that at high signal-to-noise levels $(\rho>1000)$, the reconstruction of the spectrum $f_{3}(\lambda)$ is improved compared to the case shown in Fig. 10(c). In particular, the approximation of the passband region is improved while the response in the stopband region is lowered. As $\rho$ is lowered below a critical value of approximately 100 , some spurious peaks emerge in the stopband region $(3 \mu \mathrm{m}<\lambda<6.5 \mu \mathrm{m})$. The performance degrades slightly as $\rho$ is lowered below 100 down to 0.1 . Finally, for very poor SNRs, i.e., $\rho<0.1$, the performance becomes poor in that the passband region becomes almost flat and the spurious peaks in the stopband region become amplified. Thus, the incorrect peak around 3-4 $\mu \mathrm{m}$ for a target in $8-9 \mu \mathrm{m}$ is due to an artifact by the algorithm. 


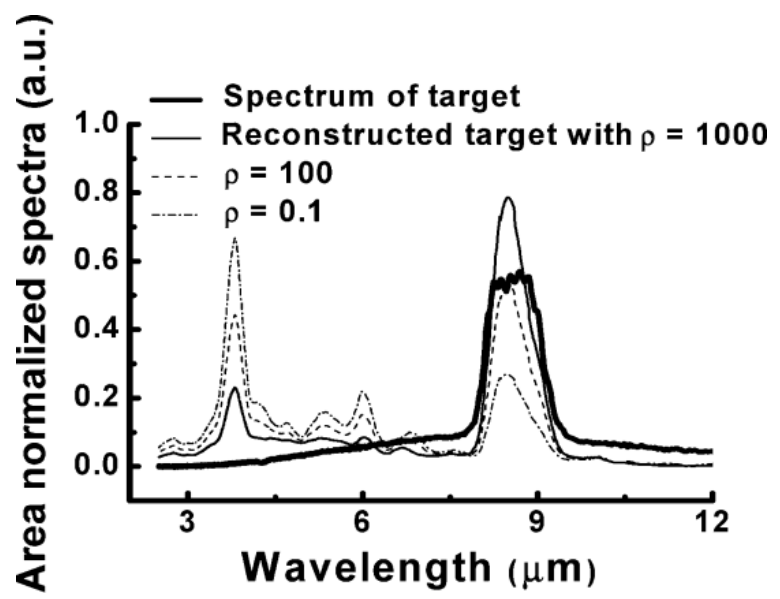

Fig. 11. Examples of experimental reconstructions of LWIR $f_{3}(\lambda)$ using algorithmic spectrometer incorporated with the DWELL-1780 detector at $30 \mathrm{~K}$ for synthesized, high $(\rho=1000)$, moderate $(\rho=100)$, and low $(\rho=0.1)$ SNRs of the photocurrent as compared to SNR of the actual photocurrent.

Next, we investigate the dependence of the performance of the algorithmic spectrometer on the DWELL's operating temperature. Here, the procedure used to generate Fig. 10(c) for the LWIR target $f_{3}(\lambda)$ was repeated for various operating temperatures of the DWELL detector and the results are depicted in Fig. 12 (dark solid curves), along with the actual spectra of the target filter (thin solid curves). As expected, the performance of the algorithmic spectrometer is degraded as the detector's temperature increases. This is a result of the increase in the dark current with temperature, which reduces the SNR as well as the overlap in the DWELL's spectral responses as the temperature increases (as it was pointed out in Section II-B). While accurate reconstruction is observed at $30 \mathrm{~K}$ [see Fig. 10(c)], as the DWELL temperature increases, the reconstructed target spectra deteriorates in the passband region. For example, the performance is poor at $77 \mathrm{~K}$. Thus, the algorithmic spectrometer at higher device temperatures cannot properly reconstruct the target peak even at 8-9 $\mu \mathrm{m}$ because at low SNRs, the peak of the DWELL detector is buried in the DWELL's noise floor [20].

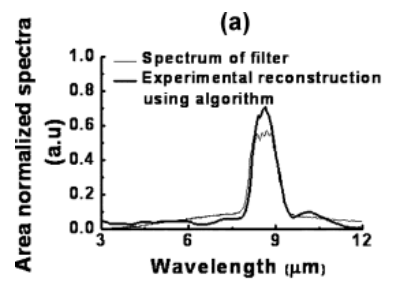

(c)

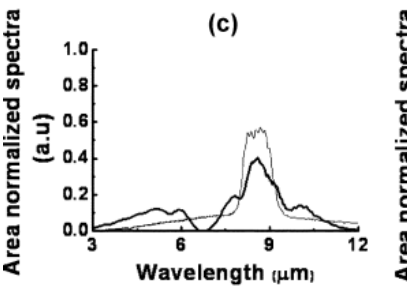

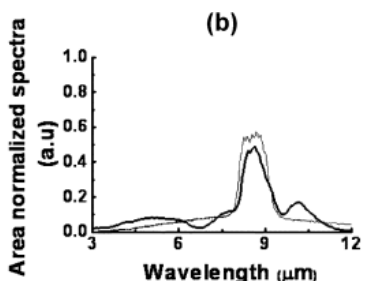

(d)

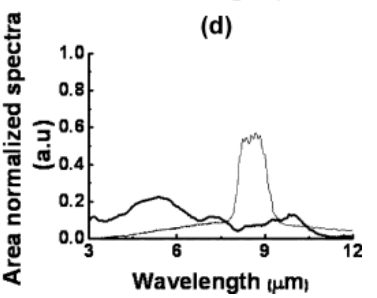

Fig. 12. Experimental reconstructions of the spectrum $f_{3}(\lambda)$ using algorithmic spectrometer incorporated with the DWELL1781 detector for various detector temperatures: (a) $30 \mathrm{~K}$, (b) $50 \mathrm{~K}$, (c) $60 \mathrm{~K}$, and (d) $77 \mathrm{~K}$. 


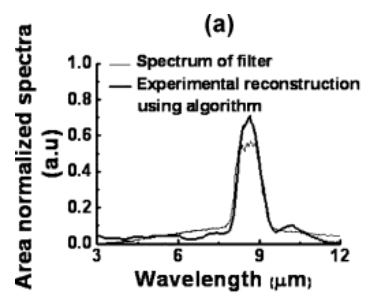

(c)

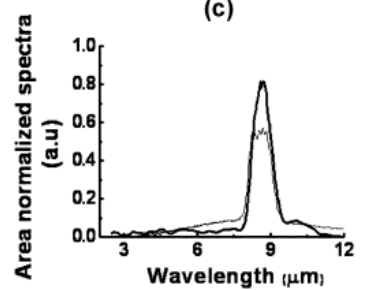

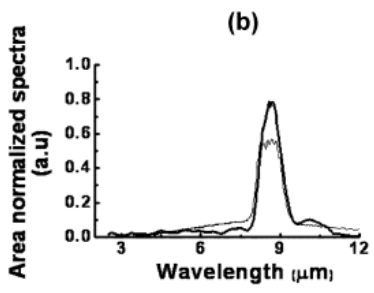

(d)

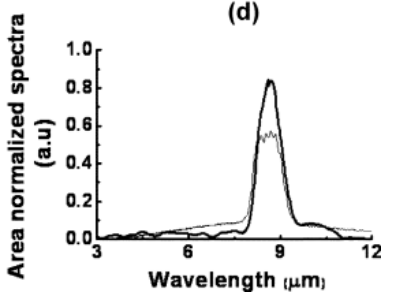

Fig. 13. Experimental reconstructions of the spectrum $f_{3}(\lambda)$ using algorithmic spectrometer incorporated with the DWELL1781 detector at $30 \mathrm{~K}$ for various bias selections: (a) with all 82 biases (from -5 to $-1 \mathrm{~V}$ in steps of $0.1 \mathrm{~V}$, and from 1 to $5 \mathrm{~V}$ in steps of $0.1 \mathrm{~V}$ ); and with a uniform subselection of (b) 40 biases, (c) 20 biases, and (d) 10 biases.

Finally, we examine the dependence of the performance on the diversity of the available operating biases of the DWELL-1781 detector. The bias selection is pursued to find the number of biases required to achieve acceptable target reconstruction. As a benchmark, all 82 bias-dependent spectra (i.e., 41 each at negative and positive biases from -5 to $-1 \mathrm{~V}$ in $0.1 \mathrm{~V}$ step, and from 1 to $5 \mathrm{~V}$ in $0.1 \mathrm{~V}$ step) of the DWELL-1781 detector were considered to generate the LWIR target $\mathrm{f} 3(\lambda)$. Then the reconstruction procedure was repeated for the subsampled biases as follows: 40, 20, and 10. Fig. 13 describes the reconstruction results for different bias selections. It was observed that a good target estimation was achieved even with many fewer number of biases (for the case of ten biases), showing the clear cutoff and strong response at the passband region. For consistency, the performances of DAS on the diversity of operating bias were further tested with the other three LWIR filter targets $f_{1}(\lambda), f_{2}(\lambda)$, and $f_{4}(\lambda)$ in Fig. 10. After applying DAS with subsampled biases, target-spectrum reconstruction was achieved for these LWIR targets with an accuracy similar to that for $f_{3}(\lambda)$. Thus, the spectral information of original target is well maintained and preserved even with a reduced number of biases. This is due to the existence of strong DWELL spectral responses at the particular bias selections. However, the selection of weak DWELL spectral responses at biases from -1.5 to $1.5 \mathrm{~V}$ leads to the poor reconstructions (results not shown).

\section{SECTION V. Conclusion}

In this paper, we demonstrated an algorithmic spectrometer comprising a DWELL detector with bias-dependent spectral response, which is due to the QCSE, and a postprocessing tuning algorithm. The implementation of the algorithmic spectrometer consists of two key stages and it requires that a target is probed repeatedly by the DWELL detector at different operating bias conditions, yielding a collection of bias-dependent photocurrents. In the first stage, sets of weights are calculated using the projection algorithm reported in [20]; in particular, one set of weights is calculated for each wavelength of interest. In the second stage and for each wavelength of interest, the photocurrents are linearly combined using the very weights associated with the specific wavelength, yielding a reconstruction of the target spectrum at that wavelength. Successful algorithmic reconstructions were obtained of the spectra of four LWIR target filters validating our approach for an algorithmic spectrometer. The performance was further examined in terms of the dependences on the photocurrent's SNR, the DWELL's operating temperature, and the diversity of the available operating biases. As expected and depending upon the DWELL's operating temperature, the performance of the algorithmic spectrometer is degraded by the increase in the dark current as the detector's temperature increases above 50 
$\mathrm{K}$ (which, in turn, reduces the SNR), and the lack of the overlap in the DWELL's spectral responses at high temperatures. The best reconstruction result was observed at $30 \mathrm{~K}$. Notably, good reconstruction can be achieved even by using only ten appropriately placed biases for which strong, overlapping DWELL spectral response exist.

Mathematical concepts behind the algorithmic spectrometer are further described here for completeness.

Each reconstructed value $\hat{I}_{\lambda_{n}}$ of the spectrum of the target of interest, at a desired tuning wavelength $\lambda_{n}$, is mathematically expressed as

$\hat{I}_{\lambda_{n}}=\sum_{i=1}^{K} w_{n, i} I_{i} \cdot(2)$

The set of weights $w_{n, i}$ for center wavelength $\lambda_{n}$, which we compactly write as $\mathbf{w}_{n}=\left[w_{n, 1}, \ldots, w_{n, K}\right]^{T}$, is given by the formula ((18) in [20])

$\mathbf{w}_{n}=\left[\mathbf{A}^{T} \mathbf{A}+\Phi+\alpha \mathbf{Q}^{T} \mathbf{A}^{T} \mathbf{A} \mathbf{Q}\right]^{-1}\left[\mathbf{A}^{T} \mathbf{r}_{\lambda_{\mathrm{n}}}\right]$ (3)

where $\mathbf{A}=\left[\mathbf{R}_{1}, \ldots, \mathbf{R}_{K}\right]$ and $\mathbf{R}_{k}=\left[R_{k}\left(\lambda_{\min }\right), \ldots, R_{k}\left(\lambda_{\max }\right)\right]^{T}$, for $k=1, \ldots, K$, while $\mathbf{r}_{\lambda_{\mathrm{n}}}=$ $\left[r\left(\lambda_{\min } ; \lambda_{n}\right), \ldots, r\left(\lambda_{\max } ; \lambda_{n}\right)\right]^{T}$. Here, the wavelengths at which the spectrum is sampled range from a minimum value of $\lambda_{\min }$ to a maximum value of $\lambda_{\max }$. Moreover, $\Phi$ is a diagonal noise-equivalent matrix whose $k$ th diagonal entry is $\mathbf{R}_{k}^{T} \mathbf{R}_{k} / \mathrm{SNR}_{k}^{2}$, where $\mathrm{SNR}_{k}$ is the SNR of the photocurrent at the $k$ th bias $v_{k}$. The regularization term $\alpha \mathbf{Q}^{T} \mathbf{A}^{T} \mathbf{A} \mathbf{Q}$ penalizes spurious fluctuations in the approximation [20]. In this paper, the matrix $\mathbf{Q}$ is taken as a Laplacian operator and $\alpha$ is the corresponding regularization weight, which is selected by the user [20]. This completes the description of the algorithmic spectrometer.

\section{References}

1. A. Rogalski, "Assessment of HgCdTe photodiodes and quantum well infrared photoconductors for long wavelength focal plane arrays", Infrared Phys. Technol., vol. 40, pp. 279-294, 1999.

2. A. Khoshakhlagh, J. B. Rodriguez, E. Plis, G. D. Bishop, Y. D. Sharma, H. S. Kim, L. R. Dawson, S. Krishna, "Bias dependent dual band response from InAs/Ga(In)Sb type II strain layer superlattice detectors", Appl. Phys. Lett., vol. 91, pp. 263504-263506, 2007.

3. S. Mallick, K. Banerjee, S. Ghosh, E. Plis, J. B. Rodriguez, S. Krishna, C. Grein, "Ultralow noise midwave infrared InAs-GaSb strain layer superlattice avalanche photodiode", Appl. Phys. Lett., vol. 91, pp. 241111-1241111-3, 2007.

4. S. D. Gunapala, K. M. S. V. Bandara, "Recent developments in quantum well infrared photodetectors" in Thin Films, New York:Academic, pp. 113-237, 1995.

5. B. F. Levine, "Quantum-well infrared photodetectors", J. Appl. Phys., vol. 74, pp. 1-81, 1993.

6. S. D. Gunapala, S. V. Bandara, C. J. Hill, D. Z. Ting, J. K. Liu, S. B. Rafol, E. R. Blazejewski, J. M. Mumolo, S. A. Keo, S. Krishna, Y.-C. Chang, C. A. Shott, " $640 \backslash \$$ times $\$ 512$ pixels long-wavelength infrared (LWIR) quantum-dot infrared photodetector (QDIP) imaging focal plane array ", IEEE J. Quantum Electronics, vol. 43, no. 3, pp. 230-237, Mar. 2007.

7. A. Rogalski, "Quantum well photoconductors in infrared detector technology", J. Appl. Phys., vol. 93, pp. 43554391, 2003.

8. S. Raghavan, P. Rotella, A. Stintz, B. Fuchs, S. Krishna, C. Morath, D. A. Cardimona, S. W. Kennerly, "High responsivity normal-incidence long-wave infrared $(\mathrm{I} ; 7.2 \mathrm{~mm}) \mathrm{InAs} / \mathrm{In} 0.15 \mathrm{GaO} .85 \mathrm{As}$ dots-in-a-well detector", Appl. Phys. Lett., vol. 81, pp. 1369-1371, 2002. 
9. J. Phillips, P. Bhattacharya, S. W. Kennerly, D. W. Beekman, M. Dutta, "Self-assembled InAs-GaAs quantum-dot inter-subband detectors", IEEE J. Quantum Electron., vol. 35, no. 6, pp. 936-942, Jun. 1999.

10. M. R. Matthews, R. J. Steed, M. D. Frogley, C. C. Phillips, R. S. Attaluri, S. Krishna, "Transient photoconductivity measurements of carrier lifetimes in an InAs/In0.15Ga0.85As dots-in-a-well detector", Appl. Phys. Lett., vol. 90, pp. 103519-1-103519-3, 2007.

11. S. Krishna, "InAs/InGaAs quantum dots in a well photodetectors", J. Phys. D (Appl. Phys.), vol. 38, pp. 21422150, 2005.

12. S. Krishna, P. Rotella, S. Raghavan, A. Stintz, M. M. Hayat, S. J. Tyo, S. W. Kennerly, "Bias-dependent tunable response of normal incidence long wave infrared quantum dot detectors", Proc. IEEE/LEOS Annu. Meeting, vol. 2, pp. 754-755, 2002.

13. S. Krishna, M. M. Hayat, J. S. Tyo, S. Raghvan, . Sakolu, Detector With Tunable Spectral Response, 2007.

14. E. S. Varley, M. Lenz, S. J. Lee, J. S. Brown, D. A. Ramirez, A. Stintz, S. Krishna, A. Reisinger, M. Sundaram, "Single bump two-color quantum dot camera", Appl. Phys. Lett., vol. 91, pp. 081120-1-081120-3, 2007.

15. E. S. Varley, D. Ramirez, J. S. Brown, S. J. Lee, A. Stintz, S. Krishna, " Demonstration of a two color 320 \Stimes\\$256 quantum dots-in-a-well focal plane array ", presented at the CLEO, 2007.

16. S. Krishna, D. Forman, S. Annamalai, P. Dowd, P. Varangis, T. Tumolillo Jr., A. Gray, J. Zilko, K. Sun, M. Liu, J. Campbell, D. Carothers, " Demonstration of a $320 \backslash \$$ times $\backslash 256$ two-color focal plane array using InAs/InGaAs quantum dots in well detectors ", Appl. Phys. Lett., vol. 86, pp. 193501-1-193501-3, 2005.

17. D. A. B. Miller, D. S. Chemla, T. C. Damen, A. C. Gossard, W. Wiegmann, T. H. Wood, C. A. Burrus, "Band-edge electroabsorption in quantum well structures: The quantum-confined Stark effect", Phys. Rev. Lett., vol. 53, pp. 2173-2176, Nov. 1984.

18. P. Aivaliotis, N. Vukmirovic, E. A. Zibik, J. W. Cockburn, D. Indjin, P. Harrison, C. Groves, J. P. R. David, M. Hopkinson, L. R. Wilson, "Stark shift of the spectral response in quantum dots-in-a-well infrared photodectors", J. Phys. D, vol. 40, pp. 5537-5540, 2007.

19. Sakolu, J. S. Tyo, M. M. Hayat, S. Raghavan, S. Krishna, "Spectrally adaptive infrared photodetectors using bias-tunable quantum dots", J. Opt. Soc. Amer. B, vol. 21, no. 1, pp. 7-17, Jan. 2004.

20. Sakolu, M. M. Hayat, J. S. Tyo, P. Dowd, S. Annamalai, K. T. Posani, S. Krishna, "A statistical method for adaptive sensing using detectors with spectrally overlapping bands", Appl. Opt., vol. 45, no. 28, pp. 7224-7234, Oct. 2006.

21. R. S. Attaluri, R. Shenoi, J. Shao, T. E. Vandervelde, A. Stintz, S. Krishna.

22. R. S. Attaluri, Growth and optimization of quantum dots-in-a-well infrared photodetectors, 2007.

23. Sakolu, Signal-processing strategies for spectral tuning and chromatic nonuniformity correction for quantumdot IR sensors, 2006.

24. P. Bhattacharya, Semiconductor Optoelectronic Devices, NJ, Englewood Cliffs:Prentice Hall, 1996. 